\title{
Understanding coalescence of phase separated droplets and verification via growing a single suspended crystal
}

\section{Zi-Qing Wu}

Northwestern Polytechnical University

\section{Yong-Ming Liu}

Northwestern Polytechnical University

Qing-Di Cheng

Northwestern Polytechnical University

Chen-Yuan Li

Northwestern Polytechnical University

Liang-Liang Chen

Northwestern Polytechnical University

Ya-Li Liu

Northwestern Polytechnical University

Wan-Yi Ge

Northwestern Polytechnical University

Sven Falke

University of Hamburg

Hevila Brognaro

University of Hamburg

Jing-Jie Chen

Northwestern Polytechnical University

Feng-Zhu Zhao

Northwestern Polytechnical University

Huan Zhou

Shanghai Institute of Applied Physics

Chen-Yan Zhang

Northwestern Polytechnical University

Xu-Dong Deng

Northwestern Polytechnical University

Ya-Jing Ye

Northwestern Polytechnical University

Wen-Juan Lin 
Northwestern Polytechnical University

Wei-Hong Guo

Northwestern Polytechnical University

Peng Shang

Northwestern Polytechnical University

Jian-Hua He

Shanghai Institute of Applied Physics

\section{Christian Betzel}

University of Hamburg https://orcid.org/0000-0002-3879-5019

Da-Chuan Yin ( $\square$ yindc@nwpu.edu.cn )

Northwestern Polytechnical University

\section{Article}

Keywords:

Posted Date: February 14th, 2022

DOI: https://doi.org/10.21203/rs.3.rs-1269229/v1

License: (1) (1) This work is licensed under a Creative Commons Attribution 4.0 International License. Read Full License 


\section{Understanding coalescence of phase separated droplets and verification via growing a single suspended crystal}

Zi-Qing Wu ${ }^{1 \dagger}$, Yong-Ming Liu ${ }^{1 \dagger}$, Qing-Di Cheng ${ }^{1,2 \dagger}$, Chen-Yuan Li ${ }^{1}$, Liang-Liang Chen ${ }^{1}$, Ya-Li Liu $^{1}$, Wan-Yi Ge ${ }^{1}$,

Sven Falke ${ }^{2}$, Hevila Brognaro ${ }^{2}$, Jing-Jie Chen ${ }^{1}$, Feng-Zhu Zhao ${ }^{1}$, Huan Zhou ${ }^{3}$, Chen-Yan Zhang ${ }^{1}$, Xu-Dong Deng $^{1}$,

Ya-Jing Ye ${ }^{1}$, Wen-Juan Lin ${ }^{1}$, Wei-Hong Guo ${ }^{1}$, Peng Shang ${ }^{1,4}$, Jian-Hua He $^{3 *}$, Christian Betzel ${ }^{*}$ \& Da-Chuan Yin ${ }^{1 *}$

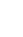

${ }^{1}$ Key Laboratory for Space Bioscience and Space Biotechnology, School of Life Sciences, Northwestern Polytechnical University, Xi'an 710072, Shaanxi, PR China

${ }^{2}$ Laboratory for Structural Biology of Infection \& Inflammation, Institute for Biochemistry and Molecular Biology, University of Hamburg, Hamburg 22607, Germany

${ }^{3}$ Shanghai Institute of Applied Physics, Chinese Academy of Sciences, Shanghai 201204, P.R. China

${ }^{4}$ Shenzhen Research Institute of Northwestern Polytechnical University, Shenzhen 518057, Guangzhou, PR China.

${ }^{\dagger}$ These authors contributed equally to this this work.

*e-mail: yindc@nwpu.edu.cn; betzel@uni-hamburg.de; hejianhua@sinap.ac.cn

Abstract: Liquid-liquid phase separation (LLPS) is a ubiquitous process found in a variety of fields. It is of particular importance in biological sciences since it plays essential and vital roles in a number of physiological and pathological processes in biological systems. After LLPS, the dense droplets can grow in size via incorporating solutes from surrounding environment and in some cases coalescing with other droplets. Interestingly, the size of the dense droplets seems to have an upper limit but the 
mechanism remains to be explored. Since the droplet size can be essential for biological functioning, it is important to understand the size evolution of the phase separated droplets. Here we propose a physical mechanism with consideration of impurities on the surface of the dense liquid droplets. The theoretical predictions can be used to explain the observations on the size evolution. Based on the coalescence mechanism, we succeeded for the first time performing a challenging task, i.e., growing a single suspended protein crystal via merging phase-separated droplets in a fully non-contact manner. The mechanism observed may be considered as a basic model for researches in much broader fields involving phase separation, such as in biology, materials science, physics, chemistry, and meteorology.

\section{Introduction}

Liquid-liquid phase separation (LLPS) in biological systems became a frontier hot topic in the field of life sciences in recent years ${ }^{1-7}$. LLPS phenomenon is ubiquitous in living organisms. For example, subcellular structures like membraneless organelles, heterochromatin, transport channel, etc, are formed through the LLPS process ${ }^{8-10}$. LLPS is also involved in a variety of important physiological processes (for examples, gene transcription ${ }^{11}$, heterochromatin formation ${ }^{8}$, DNA repair ${ }^{12}$, autophagy ${ }^{13}$, spindle assembly ${ }^{14}$, transcriptional regulation ${ }^{15}$, skin barrier formation ${ }^{16}$, noise reduction in protein concentration in cells ${ }^{17,18}$, membrane bending ${ }^{19}$, etc.), as well as a variety of pathological processes, such as Alzheimer's disease (AD) ${ }^{20,21}$, Parkinson's disease (PD) ${ }^{22}$, diabetes ${ }^{23}$, amyotrophic lateral sclerosis $(\mathrm{ALS})^{7,24,25}$, cancer ${ }^{26,27}$, leukaemic transformation ${ }^{26}$, etc. 
macromolecules and their complexes, it is often necessary to obtain their three-dimensional atomic resolution structure, through which the functions can be understood, for example via lock \& key or induced-fit models. However, this approach is not applicable for the proteins that have no distinct tertiary structure (intrinsically disordered proteins (IDPs, about $5 \%$ of total proteins in human proteome) or protein regions that are intrinsically disordered (IDRs, approcx. $55 \%$ of all proteins) ${ }^{27}$. In recent years, increasing evidences confirm that these proteins play vital roles through the LLPS process, providing a new kind of interpretation for the functions of biological macromolecules and their complexes simply via explaining thermodynamic and kinetic processes. The new perspectives can help to better understand or re-understand many physiological and pathological processes. Obviously, studying LLPS is a typical example of understanding many physiological and pathological processes from the perspective of physical sciences, and is providing an excellent bridge connecting physical sciences and biological sciences.

In investigations of LLPS in biological system, the size of the dense liquid droplets is a parameter of concern, because it can be essential for appropriate functioning ${ }^{28}$, such as the droplet size dependents of the rate of ribozyme cleavage ${ }^{29}$. Further, an interesting observation is that there seems to be an upper limit in the size of the dense liquid droplets after LLPS depending on the type and size of the cells in which they are forming ${ }^{30,31}$.

In order to understand the size evolution phenomenon, it is necessary to study the growth process of droplets. When the phase separated droplets appear in a solution, they will start to grow. Droplet growth can be achieved in two ways: 1) Capture of solutes (biological macromolecules) from surrounding solutions; 2) Collision and coalescence of the phase separated droplets. The latter is a faster way to increase the size. Here we propose a 
theoretical model for the droplet coalescence process, which can explain the phenomenon of the size limit dense liquid droplets can reach. Based on the model, we assume that continuous coalescence of dense liquid droplets can be achieved under sufficiently large external force field, and thus a single dense liquid phase can be obtained. According to the two-step nucleation theory, crystal nucleation occurs in the dense liquid phase. If a single dense liquid phase can be obtained, it is hoped that the growth of a single crystal can be realized from that single dense liquid. Based on this idea, we used the protein crystallization that starts from LLPS as a model, and successfully realized the nucleation and growth of a single, fully non-contact suspended crystal in a solution. This is a very challenging task and has never been achieved before.

Our study, on the one hand, expounds how the dense liquid droplets grow up, explains why the dense liquid droplets have an upper limit in size, and provides a new perspective for understanding the phase separation phenomenon, and the droplet size evolution in biological system. On the other hand, we have achieved a challenging task to grow single suspended crystal in solution based on this theory, suggesting that it has strong application potential in other disciplines. For example, we have found that the magnetic field influences the directional migration of dense liquid droplets. This experimental fact suggests that external physical fields can be used to influence the phase separation process, thus providing a theoretical basis for the use of physical means in the treatment of diseases. Till now there have been no reports published on the influence of external physical fields on the LLPS in biological systems, but ongoing research in our laboratory, with preliminary results, reveals an encouraging number of results in disease treatment via affecting LLPS using external physical fields. 
In addition to biological systems, phase separation and coalescence of liquid phases also occurs in other research fields, such as materials science ${ }^{32-34}$, physics ${ }^{35}$, chemistry ${ }^{36-38}$, etc. The theory proposed in this study is also useful for understanding the phenomena found in these research fields.

\section{Results}

The dense liquid phases are formed by LLPS. Theoretically, when the dense liquid droplets form, they are thermodynamically stable. Further increasing the size of the droplets is a process of decreasing the total free energy, thus it is an energy favourable process. If two dense liquid droplets are pushed together (e.g., via Brownian motion, or purposely manipulated), they may coalesce to decrease the overall free energy.

However, whether or not the coalescence occurs, depends on the status of the individual interfaces. When many impurity particles are present on the surface of the dense liquid phases, it may be difficult to realize coalescence because such impurities can prohibit direct contact of the two dense liquid phases. Here we propose a model for the coalescence of dense liquids, with consideration of the particles distributed at the interfaces between the dense liquid and the bulk solution. Fig. 1 schematically shows the model for coalescence of dense liquid droplets.

In a liquid system with two coexisting liquid phases, the interface between the two phases will have interactions with the surrounding solute and potential impurity particles to determine a contact angle $\theta$ due to the interfacial tensions ${ }^{39}$ (Fig. 1a). When the contact angle $\theta=0^{\circ}$, the impurity particles will be completely "wetting" on the interface, so that the particle will tend to stay inside the dense liquid phase. While when the contact angle $\theta=180^{\circ}$, the 
impurity particles will be completely "super-hydrophobic" at the interface, so that the

2 particles will be more likely to stay in the bulk solution. If $\theta$ does not equal to either $0^{\circ}$ or

$3180^{\circ}$, the interface will tend to capture the impurity particles. In summary, the status of the

4 impurity particles at the interface will depend on the contact angle $\theta \quad$ (Fig. 1a).

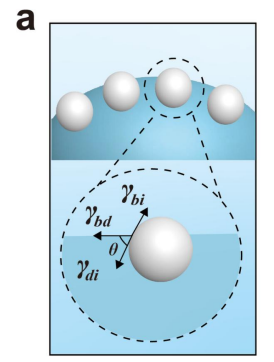

b

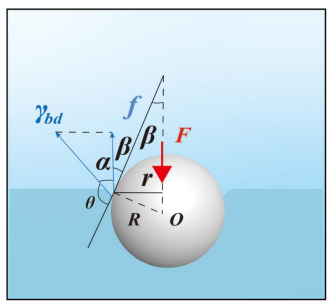

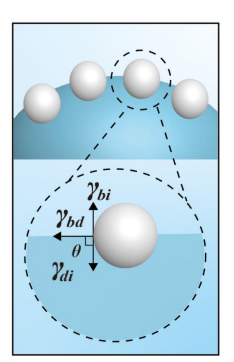

C
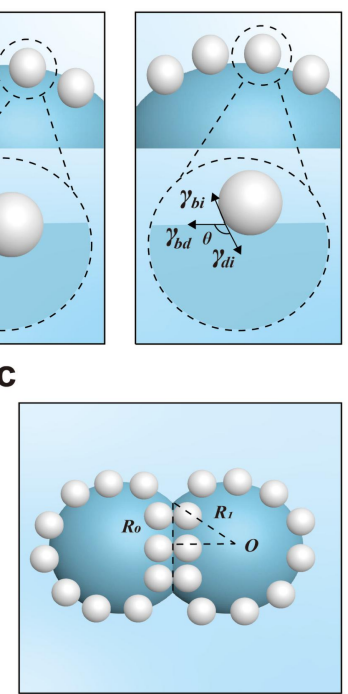

d

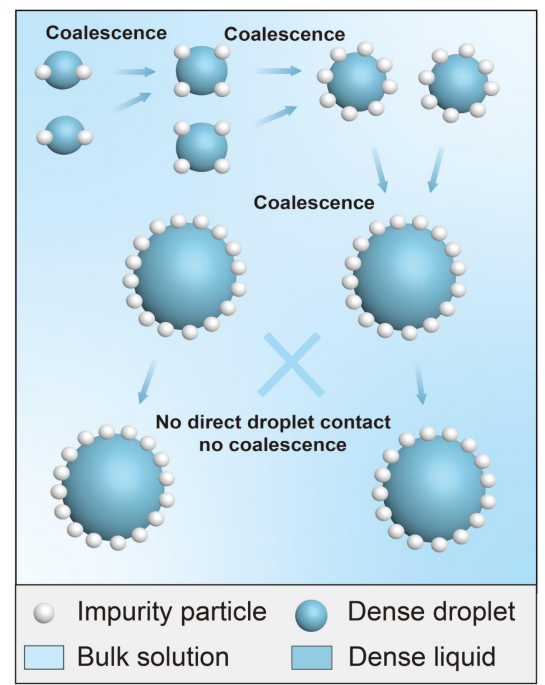

Fig. 1| Theoretical model of phase coalescence of dense liquid droplets. (a) Different status of the impurity particles captured by the interface between the dense liquid and the bulk solution. If the contact angle $\theta$ (depending on the interfacial tensions $\gamma_{d i}, \gamma_{b d} . \gamma_{b i}$ ) is not equal to $0^{\circ}$ or $180^{\circ}$, the impurity particles tend to be captured by the interface. (b) A close look at the interfaces between the dense liquid, bulk solution and impurity particles. Force $F$ is used to push the impurity particles into the dense liquid droplet. (c) Two dense liquid droplets pushed together, with impurity particles pushed into the droplets so that the dense liquids can be in direct contact with each other. (d) Different stages of coalescence of dense liquid phases. The density of the impurity particles on the droplet surface will increase upon each coalescence, making it more and more difficult to coalesce further.

To realize coalescence of two dense liquid droplets, direct contact of the dense liquid 
1 phases is necessary. When there are impurity particles at the interface, a force $F$ is

2 indispensable to "push" the impurity particles at the interface into the dense liquids (Figs. 1b,

3 1c) allowing the two dense liquid phases to get in direct contact with each other. Based on the

4 balance between the interfacial tension and the force $F$, we derived the following equation

5 (Eq. (1), see Supplementary Derivation 1 for detailed derivation):

$$
F \geq \mathrm{n} \pi^{2} R R_{0}^{2} \gamma_{b d}(1-\cos \theta),
$$

where $n$ is the density of impurity particles (i.e., number of particles in a unit area at the interface), $R$ is the radius of the impurity particle, $\gamma_{b d}$ is the interfacial tension between the bulk solution and the dense liquid, $\theta$ is the contact angle between the dense liquids and the impurity particle, and $R_{0}$ is the minimum radius of the contact area between two dense liquid phases when the impurity particles are "pushed" into the dense liquid phases.

We have noticed that, in each coalescence, the density of the impurity particles $(n)$ at the interface will increase if the total amount of the impurity particles at the interfaces is assumed to be unchanged. Given that the initial density is $n_{0}$, the density after one coalescence is $n_{1}$, and the density after coalescence for $m$ times is $n_{m}$, the following equations can be derived:

$$
n_{m}=2^{\frac{2}{3} m} n_{0} \text {. }
$$

In the theoretical model there are impurity particles distributed at the interfaces. In an ideal solution, suppose there are no impurity particles, i.e., $n=0$, then the force necessary for a successful coalescence shall be $F \geq 0$, which means that no or very weak external force is necessary to achieve the coalescence.

Eq. (2) shows that, for each coalescence, the density of the impurity particles at the interface will increase by $58.7 \%\left(2^{2 / 3}=1.587\right)$. It means that the density will sharply increase after a number of coalescences. If the density is very high, the force necessary for the 
coalescence will be high, so that it may be practically impossible to coalesce further repeatedly (Fig. 1d). Hence we can understand why the dense droplets exhibit limited size, as reported in previous publications ${ }^{30,31}$. In reality, the environment in the living cells is rather crowded with numerous different species of macromolecules, small molecules, ions, different fragments, particles, etc. These randomly moving species can be captured by the surface of the dense liquid droplets, increasing the impurity particle density on the surface, thus blocking the incorporation of more macromolecules into the droplets, and causing difficulties in droplets coalescence. This scenario may be a partial reason why aging droplets showed difficulty to coalesce ${ }^{12}$, because aging droplets have more time to capture more impurities from the surrounding environment.

According to the above mechanism, if we reduce the density of the impurities, or increase the force for coalescing the droplets, we might be able to obtain a single large drop in the solution. This scenario is quite intriguing because we can immediately think of using protein crystallization as a model to test the assumption. It is well-known that LLPS occurs before nucleation of protein crystals, as described in the two-step nucleation theory ${ }^{40-42}$. In a protein crystallization solution, if we can merge all the phase separated droplets to form a large one, we may be able to obtain a single suspended protein crystal in the solution from that drop, in a fully non-contact manner, which has never been achieved before.

In order to test the above concept, we used a gradient magnetic field as a tool to non-contactly move and merge dense liquid droplets. To observe if magnetic fields can manipulate dense liquid phases, we applied permanent magnets on a model solution containing phase separated protein droplets. Since the product $B B^{\prime}$ of magnetic field $B$ and field gradient $B^{\prime}$ provided by a permanent magnet is low (less than $40 \mathrm{~T}^{2} / \mathrm{m}$ for a cylindrical 
$1 \mathrm{NdFeB}$ magnet with dimensions $\phi 35 \mathrm{~mm} \times 30 \mathrm{~mm}$ ), the magnetic force $F_{\mathrm{m}}$, which is 2 proportional to $B B^{\prime}$, will be small when the solution conditions are fixed. To increase the 3 magnetic force, the difference in the magnetic susceptibility $\Delta \chi$ between the dense liquid and 4 the bulk solution shall be large enough, which can be realized by adding a paramagnetic salt to the protein solution. In this study, the paramagnetic salt $\mathrm{MnCl}_{2}$, which exhibits high

6 magnetic susceptibility, was used.
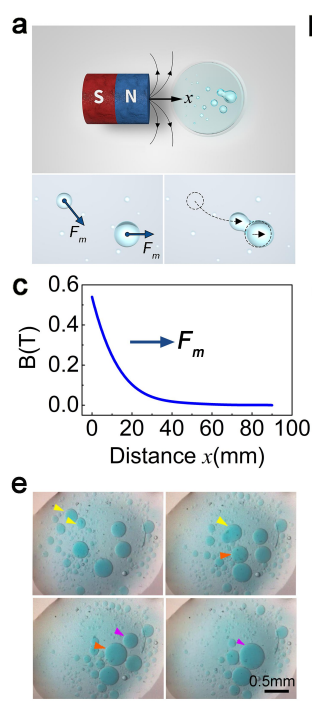

g

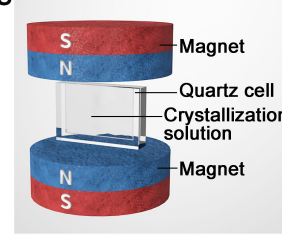

$\mathrm{h}$
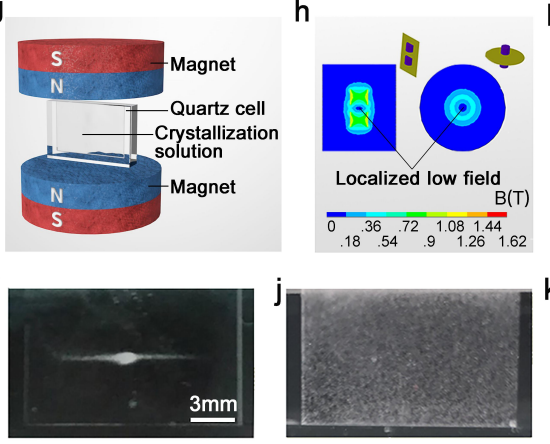

$\mathrm{m}$

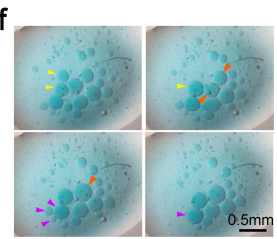

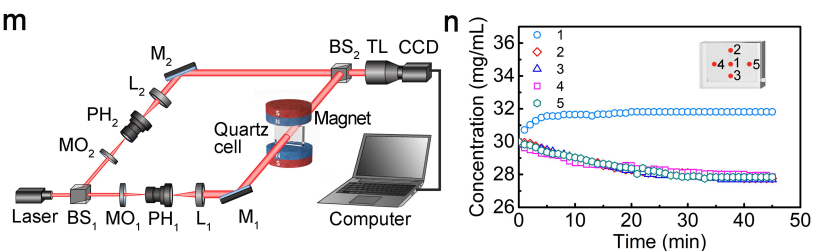

Fig. 2 | Evidence of non-contact manipulation of dense liquid droplets using gradient magnetic

field. (a) Schematic illustration of the dense liquid droplets pushed away non-contactly by a magnet.

The droplets may merge together under the influence of the magnetic force $F_{m}$. (b) Schematic illustration of the dense liquid droplets pushed to the central zone non-contactly by two opposite magnets. (c) Magnetic field distribution along the axial direction from the cylindrical magnet (corresponding to (a)). The arrow shows the movement direction of the droplets, which is towards the low field zone. (d) Magnetic field distribution along the axial direction between the two cylindrical magnets (corresponding to (b)). (e) Experimental observation of phase coalescences under the influence of a cylindrical magnet as in (a). The arrows indicate the droplets that are merged together subsequently. (f) Experimental observation of phase coalescences under the influence of two opposite 
magnets arranged as in (b). (g) The structure of an N-N permanent magnet pair with assembling

2 frameworks and a quartz sample cell. The quartz cell was placed between the N-N permanent magnet

3 pair. The quartz cell has a thin layered space filled with protein crystallization solution. (h) The

4 magnetic field distribution in the N-N permanent magnet pair as in (g). (i) Localized crystallization of

5 protein in a Galaxy-shaped zone which is coincident with the low magnetic field zone (as in (h)),

6 showing that the dense liquid droplets from which the crystals are formed were pushed to the low

7 magnetic field region. (j) Crystallization without magnetic field. The crystals randomly distributed

8 everywhere in the cell. (k) Localized lysozyme crystallization at the site with lowest magnetic field,

9 when the initial supersaturation level is lower than the case in (i). (l) A zoomed-in image of the

10 lysozyme crystals formed in (k). (m) The quartz cell with a thin layered solution between two magnets

11 is placed in the optical path of a Mach-Zehnder interferometer that records the evolution of the

12 interference images which can be transformed to the evolution of solution concentration. (n) The

13 concentration distribution was measured using the interferometer. The lysozyme concentration at the

14 site (point 1) with the lowest magnetic field is higher than in the surrounding environments (points 2-5), proving that the dense liquid droplets were indeed pushed to the lower magnetic field zone.

By placing a cylindrical permanent magnet at one side of a solution with phase-separated dense liquid droplets (Fig. 2a, Supplementary Movie 1), we clearly observed a movement of droplets away from the permanent magnet, in the direction to the low magnetic field. Fig. $2 \mathrm{c}$ shows the magnetic field distribution in the axial direction of the permanent magnet, from

21 which the force direction (also the movement direction of the dense liquids) is indicated. Fig. 22 2e (more in Extended Data Fig. 1) presents a series of images, showing that the droplets are pushed away from the permanent magnet. If we place another permanent magnet at the 
1 opposite side of the solution, with the same magnet pole (north pole, $\mathrm{N}$ pole) facing right to

2 that of the previous one (N-N poles, Fig. 2b), the movement of the droplets to the center

3 (where the magnetic field is the lowest, Fig. 2d) of the two magnet poles can be observed

4 (Supplementary Movie 2). Fig. 2f (more in Extended Data Fig. 2) shows a series of images of

5 this process. The dense liquids were pushed together to the region with localized low

6 magnetic field, and coalescence of some dense liquids occurred (indicated by the arrowheads),

7 demonstrating that the magnetic force can be used to non-contactly manipulate the dense

8 liquid droplets and actively trigger the coalescence of the droplets in the phase separated

9 protein solution.

10 As illustrated in the section above, permanent magnets can push the dense liquid droplets together and can cause coalescence. This phenomenon indicated that, if the crystals nucleate from the dense liquid droplets, it will be feasible to control the location and number of the final crystals. To examine if it is possible to control the nucleation site, we designed an apparatus with an N-N permanent magnet pair, i.e. a pair of permanent magnets with their $\mathrm{N}$ poles facing right towards each other, and a quartz cell with a thin layer of protein crystallization solution placed between the N-N poles (Fig. $2 \mathrm{~g}$ ). The geometrical arrangement of the permanent magnets created a localized low magnetic field between the two $\mathrm{N}$ poles (Fig. 2h).

In the case when the quartz cell was placed in the magnetic field (Fig. 2g), the crystals would appear at distinct positions. According to the results of non-contact manipulation of 21 dense liquids using permanent magnets (Figs. 2e-f), we expected that the crystals would appear at the localized low field zone. Our experimental observation confirmed this expectation (Figs. 2i-1). It can be seen that the crystals appeared in the localized low field 
zone (Fig. 2i), with a shape resembling the side view of the Galaxy, indicating that the dense liquids were pushed to the localized low magnetic field and crystals then formed from the dense liquids. As the initial concentration decreased, the number of crystals decreased, and the crystals appeared at the site with the lowest magnetic field (Figs. 2k-1). Fig. 21 shows the zoomed-in image of the localized crystals in Fig. $2 k$. The crystals can be seen to orientate along the vertical magnetic field direction. Compared with the localized crystallization in the magnetic field, the crystals appeared everywhere in the cell (Fig. 2j) when the quartz cell was placed outside of the magnetic field.

Concentration measurements using a Mach-Zehnder interferometer (Fig. 2m) also showed that the concentration at the localized low magnetic field strength zone (Fig. 2n, Point 1) increased, while the concentration in the surrounding environments decreased (Fig. 2n, Points 2 - 5). This is another strong evidence supporting that the dense liquid droplets were non-contactly pushed together to the localized low magnetic field zone.

To further increase the magnetic force or $B B^{\prime}$, so as to achieve a more complete coalescence of dense liquids, we used a large gradient magnetic field provided by a superconducting magnet. Fig. 3 shows a protein crystallization experiment using a large gradient high field superconducting magnet. Fig. 3a exhibits the overall schematic diagram of the superconducting magnet experimental platform (detailed configuration of the superconducting magnet can be found in Extended Data Fig. 3). The crystallization experiment was carried out in a sample container which was placed in the magnet bore. Fig. $3 \mathrm{~b}$ shows schematic illustration of the sample container, with a force analysis on a non-contact suspended crystal in the solution. The forces acting on the suspended crystal include the gravitational force $(G)$, the buoyancy force $\left(F_{b}\right)$, and the magnetic force $\left(F_{m}\right)$. 
1 Theoretically, if the gravitational force is counterbalanced by buoyancy and magnetic forces,

2 the crystal can be non-contactly suspended in the solution. To monitor the crystallization

3 process, two CCD cameras were used to capture the top-view and side-view images of the

4 sample solution (Fig. 3a), to provide solid evidence for non-contact crystallization.

5

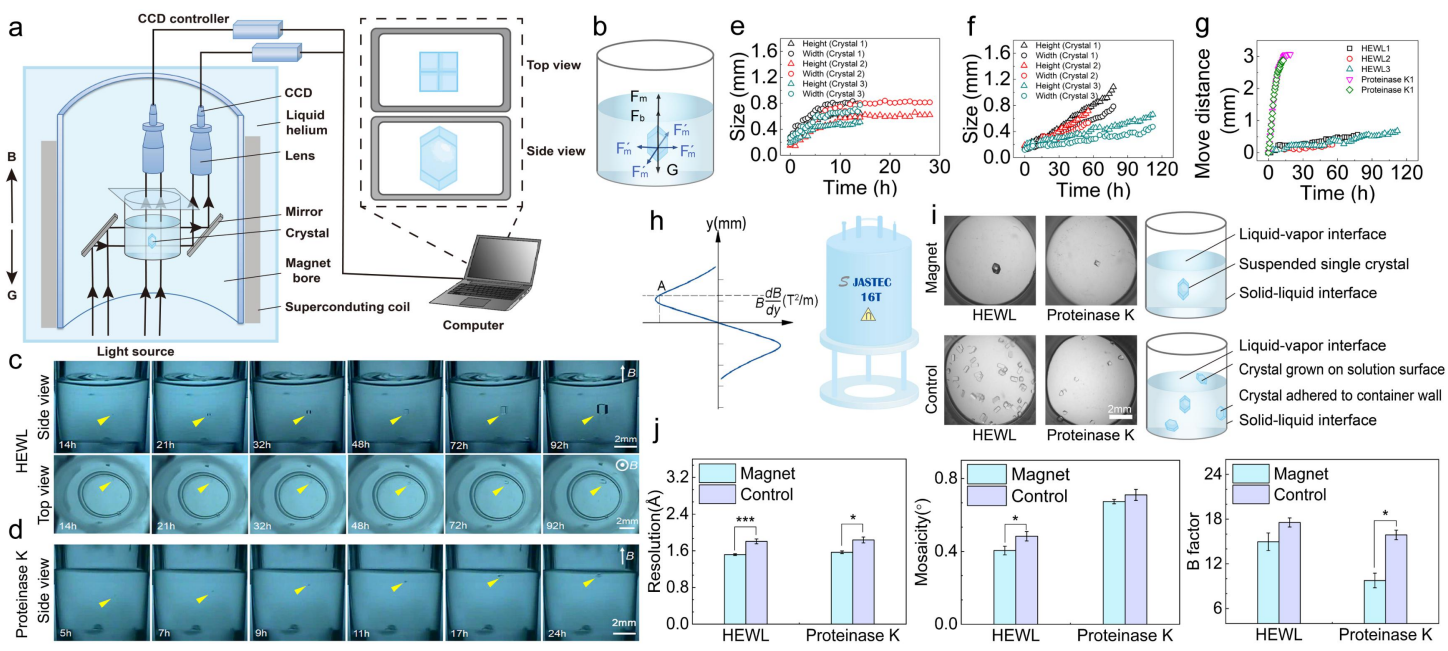

Fig. 3| Nucleation and growth of a suspended single protein crystal in a superconducting magnet.

(a) Illustration of the magnet platform, including the superconducting magnet, crystallization unit, observation and illumination systems. (b) The crystallization cell and the forces (magnetic force $F_{m}$, buoyancy force $F_{b}$, and gravitational force $G$ ) acting on the suspended crystal. The horizontal forces are magnetic forces which are cancelled in the symmetrical field. (c) Time course side-view images (upper row) and top view images (lower row) of non-contact nucleation and growth of HEWL crystal at 14, 21, 32, 48, 72 and 92 hours after starting the experiment. (d) Time course side-view images of non-contact nucleation and growth of proteinase $\mathrm{K}$ crystal at 5, 7, 9, 11, 17 and 24 hours after starting the experiment. (e) Size evolution of the crystals grown in normal conditions after the crystals were larger than $0.1 \mathrm{~mm}$. (f) Size evolution of a single suspended crystals grown at non-contact conditions after the crystals were larger than $0.1 \mathrm{~mm}$. (g) Time dependence of the ascending distance of the suspended protein crystals. The zero position was set at the site where the crystal size is larger than $0.1 \mathrm{~mm}$. (h) 
1 The superconducting magnet and its axial distribution of the magnetic field. (i) Protein crystals grown

2 at suspended and normal conditions. Left column: Typical crystal images obtained in suspended and

3 normal conditions. Right column: schematic illustration of the crystals obtained in different

4 environments. (j) Quality comparisons of the crystals obtained in suspended and normal conditions.

5 The crystals obtained in suspended conditions show superior quality to the control. Left: Max.

6 resolution; Middle: mosaicity; Right: overall B factor.

7

Two proteins (hen-egg white lysozyme and proteinase K) were used as model proteins. In the crystallization process of lysozyme, a visible suspended single crystal (size: $\sim 0.1 \mathrm{~mm}$ ) appeared approximately 11-24 hours after starting the experiment. During the whole growth process, the suspended crystal appeared to remain motionless in all experimental runs. Fig. 3c shows series of images of a typical non-contact crystallization experiment of lysozyme. In this experiment, we recorded both the side- and top-view videos of the crystallization process for about 92 hours. The crystal first became observable (from side-view, Fig. 3c, when the crystal size was $0.1 \mathrm{~mm}$ ) after about 14 hours. The videos can be shortened for easier observation (Supplementary Movie 3). More repeated experiments were performed, Supplementary Movie 4 exhibited one more example of non-contact crystallization of lysozyme, which was carried out for about 48 hours considering the crystal size requirement for the X-ray diffraction analysis. Six times repeated experiments confirmed the same phenomena. Hence we realized the non-contact crystallization of a suspended lysozyme crystal using the directed magnetic force which pushed the dense liquid droplets together to form a large one, and subsequently a single suspended crystal formed from that drop. 
1 Supplementary Movie 6 provides an animation movie to show the process. As a comparison,

2 Supplementary Movie 7 shows an animation of crystallization under normal conditions.

Figs. $3 \mathrm{e}$ and $3 \mathrm{f}$ show the size evolution of lysozyme crystals grown in different conditions.

In the case of crystal growth in standard conditions (Fig. 3e), the crystals grew fast in the beginning, after the crystals were visible, and stopped growing after several hours. In the case of non-contact crystallization (Fig. 3f), it can be seen that the crystal size is linearly increasing against the time after the crystal became visible, showing that the growth rate was constant during the observation period. The constant growth rate indicated that the supersaturation around the crystal was constant, which is a further indication that a solute depletion zone was formed around the crystal. This is a good evidence to show, that the convection in the solution was low or damped under such non-contact crystallization condition. Furthermore, the much slower growth rate in the non-contact crystallization experiment, compared with the normal case, is also in accordance assuming rather low convection.

In the case of proteinase $\mathrm{K}$, crystallization of a non-contact single crystal was also successfully achieved. Strikingly different from the seemingly motionless non-contact crystallization of lysozyme, the suspended crystal of proteinase $\mathrm{K}$ keeps moving upward during the entire growth process. For this model protein, the suspended proteinase $\mathrm{K}$ crystal appeared about 5-10 hours after starting the experiment, and lasted for about 24 hours throughout the whole crystallization experiment. Fig. 3d shows one example of the time-course evolution of proteinase $\mathrm{K}$ crystal growth from side-view. In this example, the crystal appeared at about 5 hours after starting the experiment. The recorded video was cut into a shortened version (Supplementary Movie 5), from which the growth and ascending 
1 process can be clearly observed. Fig. $3 \mathrm{~g}$ shows the moved ascending distance against the time

2 since the crystal became visible from the side-view CCD camera. It can be seen that the

3 proteinase $\mathrm{K}$ crystal kept ascending and the movement was faster in the earlier period, and

$4 \quad$ later the crystal movement slowed down.

5 As mentioned above, we have observed significantly different crystallization behaviours

6 of the two proteins. In the case of lysozyme crystallization, we found that the crystal appeared

7 to remain motionless during the entire growth process, while in the case of proteinase $\mathrm{K}$, the

8 crystal kept moving upward during the growth process, although the movement was rather

9 slow (approximately $2.16 \mathrm{~mm}$ in one day). Apparently, the movement of proteinase $\mathrm{K}$ crystals

10 indicated that the forces acting on the crystal changed during the growth process.

11 To identify the reason why the crystals of the two proteins behave so differently, we derived the requirement (Eq. (3), refer to the Supplementary Derivation 2 for detailed derivation) for keeping the crystals motionless based on the force balance in the vertical direction (Fig. 3b).

$$
\frac{\left(\chi_{c r y s t}-\chi_{\text {sol }}\right)}{\left(\rho_{\text {cryst }}-\rho_{\text {sol }}\right)}=\frac{g \cdot \mu_{0}}{B \frac{d B}{d y}}=C,
$$

where: $\chi_{\text {sol }}$ and $\chi_{\text {cryst }}$ are the magnetic susceptibility of the solution and the crystal, respectively; $\rho_{\text {sol }}$ and $\rho_{\text {cryst }}$ are the density of the solution and the crystal, respectively; $B$ is the magnetic field, and $d B / d y$ is the field gradient in the vertical direction (y), $g$ and $\mu_{0}$ are the gravitational acceleration and the magnetic permeability, respectively, which are both constants. $C$ is also a constant.

From Eq. (3), it can be seen that, if the density of the bulk solution $\left(\rho_{\text {sol }}\right)$ changes, the 
susceptibility $\left(\chi_{\text {sol }}\right)$ must change accordingly to ensure that the equation is correct. However it would be difficult to vary $\chi_{\text {sol }}$ exactly with varying $\rho_{\text {sol }}$ to make the constant $C$ unchanged. In other words, it shall be difficult to keep the growing crystal motionless in the solution.

Based on the theoretical analysis, we did more careful analysis of the position of the growing lysozyme crystal based on the image series obtained, and found that indeed, though not obvious, the growing lysozyme crystal was moving upward. The movement was approximately $0.40 \mathrm{~mm}$ in 92 hours, which was far slower than the movement of the proteinase K crystal (Fig. 3g).

The movement distance of the proteinase $\mathrm{K}$ crystal is much larger than that obtained for the lysozyme crystal. Such difference indicated that either $\chi$ sol or $\rho_{\text {sol }}$ changed more significantly in the case of proteinase $\mathrm{K}$ than in the case of lysozyme. To obtain more insights about the involved mechanism we solved the crystal structure of both proteins. Extended Data Fig. 4 shows the crystal structures of the two proteins. We noticed that, there are $2 \mathrm{Ni}^{2+}$ ions and $4 \mathrm{Cl}^{-}$ions bound to each lysozyme molecule. While in the case of proteinase $\mathrm{K}$, no bound $\mathrm{Ni}^{2+}$ ion was detected. It means that, the overall amount of $\mathrm{Ni}^{2+}$ ions is less in the proteinase $\mathrm{K}$ crystals than in the lysozyme crystals. Since $\mathrm{Ni}^{2+}$ is paramagnetic, less $\mathrm{Ni}^{2+}$ ions in proteinase $\mathrm{K}$ crystals means that the proteinase $\mathrm{K}$ crystal is "more diamagnetic" than the lysozyme crystal. According to the force balance on the crystal (Fig. 3b), $F_{m}$ shall be larger for proteinase $\mathrm{K}$ than for lysozyme. Therefore, the combined force $F$ is larger in the case of proteinase $\mathrm{K}$, resulting in a more evident movement during crystallization of proteinase $\mathrm{K}$, than in the case of lysozyme.

During non-contact crystallization, the crystal is suspending in the solution. Therefore, the 
1 growth of the crystal is different from that in conventional crystallization, as in the latter case

2 the crystal is in contact with a certain surface/interface, so the growth will be limited by the

3 surface and interface. While in the non-contact crystallization, the crystal has the freedom to

4 grow in any direction, the crystal obtained by non-contact crystallization shows an "unbiased"

5 morphology, with all crystal facets fully developed. Fig. 3h shows the magnetic field

6 distribution of the superconducting magnet. Fig. 3i shows the difference of the crystals and

7 the growth environments between the non-contact crystallization with the superconducting

8 magnet (Fig. 3i, upper) and the normal crystallization in contact with a container wall without

9 that magnet (Fig. 3i, lower). Crystallization at the liquid/air interface will also limit the

10 growth, so that a crystal with "unbiased" morphology is difficult to obtain. Therefore,

11 non-contact crystallization occurs totally in the solution and can be considered as an ideal

12 condition for obtaining high quality crystals.

13 The quality of the crystals obtained from the non-contact crystallization is compared with those obtained from the normal crystallization, and an obvious enhancement in crystal quality by using the non-contact crystallization was confirmed. In total, six experiments have been repeated for growing HEWL single crystals, and three experiments have been repeated for proteinase K. The highest resolution obtained for the crystal grown via the non-contact crystallization reached $1.45 \AA$ for HEWL and $1.51 \AA$ for proteinase $\mathrm{K}$ (using an in-house fixed target X-ray source), while that from the normal crystallization reached $1.69 \AA$ for HEWL and $1.75 \AA$ for proteinase K (Fig. 3j, left). Mosaicity (Fig. 3j, middle) and overall B factors (Fig. 3j, right) for both proteins also confirmed the same tendency (Detailed X-ray crystallography data are available in Supplementary Tables 1-5). The enhancement of crystal quality by using 
1 a magnetic field has been reported and the mechanisms have been studied before ${ }^{43}$. The

2 proposed mechanisms include the orientation effect in a magnetic field and damped

3 convection in the magnetic field. In this work described here, the crystals grown by the

4 non-contact crystallization have the additional benefit that the crystal growth is not limited by

5 any surfaces/interfaces, so that there will be no internal "stress" arising from the lattice

6 restriction due to the contact with the surfaces / interfaces.

7 Hence, based on the theoretical model of phase coalescence, we have achieved growing a

8 single suspended protein crystal in a fully non-contact manner. Realization of the challenging

9 task demonstrated the application potential of the model, which we believe is useful in several

disciplines (Fig. 4), including investigations of LLPS in both biological and physical sciences.

In the field of protein crystallization, there are some enigmatic phenomena. Here we will explain two of them using the model proposed in this work. It was found that the concentration of the protein in the crystallization solution is increasing over time at the lower

14 side of a crystallization container, and simultaneously it is decreasing at the upper side ${ }^{44}$. This phenomenon was not explained in the past, but now we understand that the phenomenon is caused by phase coalescence. In the crystallization solution, phase separated droplets are moving randomly due to Brownian motion, which will lead to many collisions between the droplets. When two droplets collide with each other, coalescence may occur so that the

19 resulting droplets become larger. When the droplets become large enough, they will sediment to the bottom of the reservoir, resulting in an increase of the protein concentration at the bottom, before crystals are forming. Simultaneously, the concentration at the upper side of the container will decrease. 


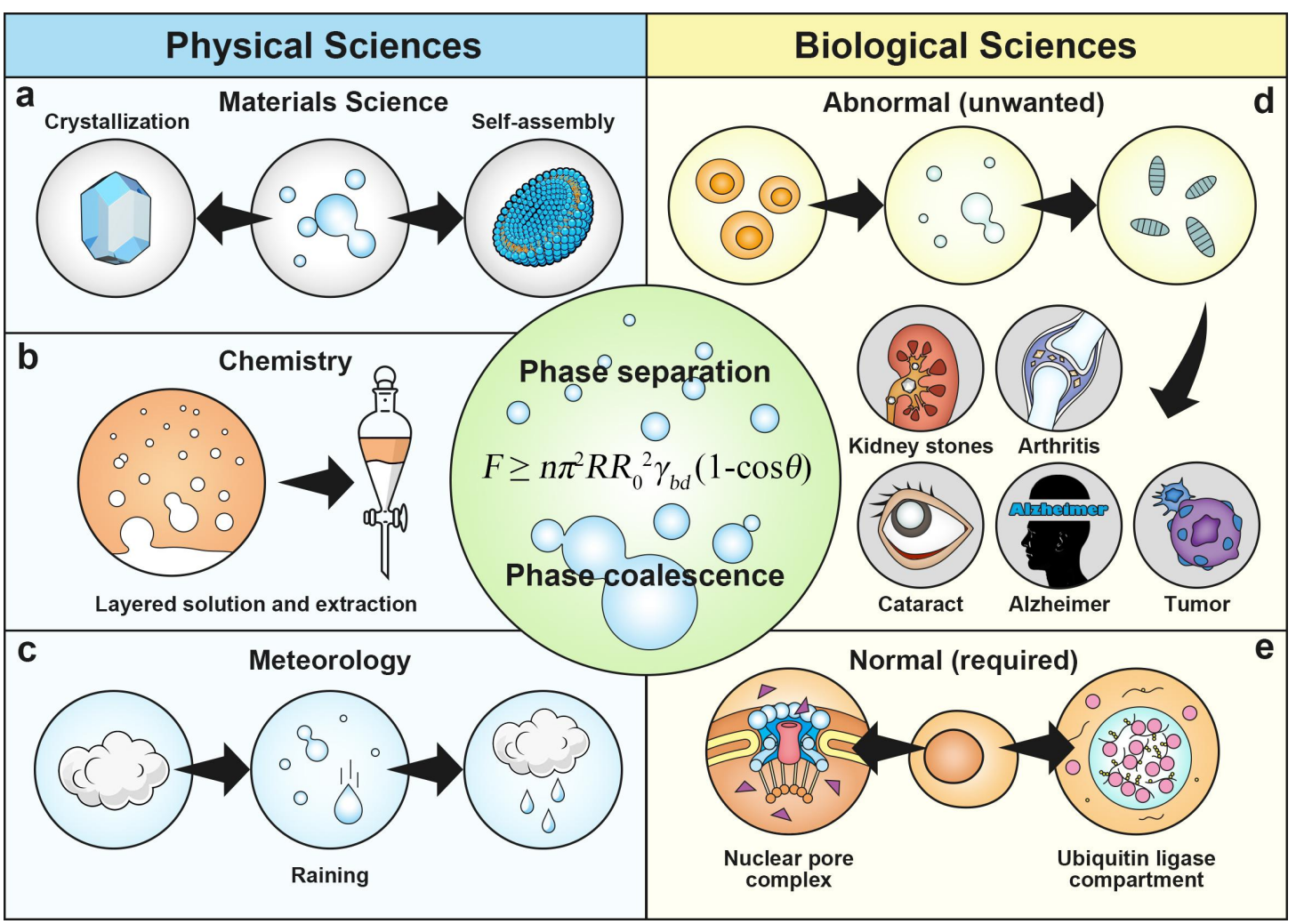

2 Fig. 4 The model of phase coalescence is applicable in many disciplines. Separation and

3 coalescence of liquid phases are often observed in the fields of physical and biological sciences. The

4 figure shows examples in different disciplines, where the phase coalescence theory can be applied.

5 Phase separation and coalescence are fundamentally important in many processes, such as: (a)

crystallization or self-assembly in materials science; (b) extraction in chemistry; (c) rain formation in

Another observation, which surprised crystallographers for many years, can be also explained using the phase coalescence model. For some proteins, no matter how to fine-tune the crystallization conditions, one may obtain either a clear drop or a drop containing numerous small crystals. It seems that there is no way to get a small number of large crystals. 
1 According to the coalescence model, we believe that, the density of the impurity particles

2 might be high, so that coalescence events are not easy to achieve. Since a successful

3 crystallization requires that the size of the dense liquid droplets is sufficiently large, small

4 sized dense liquid droplets might not be able to form ordered nuclei, or small nulcei are

5 formed but they do not grow due to the existence of the impurities on the surface, so that the

6 crystallization solution appear clear. However, when the size of the dense liquids becomes

$7 \quad$ slightly larger, numerous small crystals will be observable.

8 In addition to the context of protein crystallization, the coalescence of dense liquids is also

9 frequently observed in other processes, and Eqs. (1) and (2) are useful to explain the 10 phenomena in those processes.

11 According to Eq. (1), in an ideal solution system when there are no impurities present $(n=$

$120)$, or the impurities are completely immersed into the bulk solution $\left(\theta=180^{\circ}\right) /$ or the dense

13 liquids $\left(\theta=0^{\circ}\right)$, the dense liquids will coalesce after phase separation, resulting in layered

14 solutions in the gravitational field. Similarly, in the nature environment, when the concentration of dust particles is nearly zero in regions with high humidity, it will be easier to trigger raining via stimulation like audible sound. Therefore, in a region where the rain can be produced by shouting or clapping hands, the air in that region is indicated to be very clean.

18 This phenomenon was reported as a "mysterious attraction", e.g at the Listening Lake (Tingming Lake or Miren Lake) in Yunnan Province in China.

In the biological systems, LLPS is now a well-known phenomenon which is found to be 21 essentially involved in many physiological and pathological processes, as described in the 22 beginning of this article ${ }^{1-7}$. According to Eq. (1), the coalescence of dense liquids is related 23 with parameters $n$ (the density of impurities), $R$ (the size of the impurity), $\gamma_{b d}$ (the interfacial 
1 tension between the bulk solution and the dense liquid), and $\theta$ (the contact angle between the

2 dense liquid and the impurity). Therefore, the coalescence is affected by the density of

3 impurities $(n)$, the size of the impurities $(R)$, and the material types, structures and properties

4 of the three phases (impurity, dense liquid, and bulk solution), which determine the

5 parameters $\theta$ and $\gamma_{b d}$. According to this basic equation, one may theoretically foresee some

6 processes or explain the phenomena occurring in living cells during LLPS, like the examples

$7 \quad 12,45$ explained before. Once rather large dense liquid droplets are observed, this may indicate

8 that the coalescence occurs fast, or the impurities in the cell, i.e. physiological products,

9 different particulate species, and other fragments, are low in quantity. In context of liquid

10 dense cluster formation more biological processes need to be studied, to provide more

11 insights about LLPS processes in the biological systems.

\section{Conclusions}

In this paper, we presented a theoretical model of phase coalescence to understand the interesting phenomenon that the phase separated droplets after LLPS in biological system has an upper limit in size. Based on the theory, we proposed a new approach using protein crystallization as a model, and successfully achieved the nucleation and growth of a single suspended crystal in the solution, which is a quite challenging task and has never been realized before. Because LLPS plays very important roles in physiological and pathological processes in biological systems, a better understanding of the physical process of LLPS is of

21 high value for related studies. The model presented in this study meet this requirement and 22 can be very helpful in further studies. Furthermore, a very important implication can be inferred from the experimental fact that external physical fields can affect LLPS process. In 
1 other words, the phenomenon can provide theoretical basis for the utilization of external

2 physical fields in influencing LLPS and hence in treatments of LLPS related diseases.

3 In research fields other than biological sciences, LLPS is also a ubiquitous phenomenon

4 and phase coalescence occurs in many chemical processes. The theoretical model proposed in

5 this study can therefore be also useful for studying LLPS processes in these fields (materials

6 science, physics, chemistry, meteorology, etc.). 


\section{References}

2 1. Brangwynne, C. P. et al. Germline P granules are liquid droplets that localize by controlled

3 dissolution/condensation. Science 324, 1729-1732 (2009).

4 2. Alberti, S., Gladfelter, A., \& Mittag, T. Considerations and challenges in studying liquid-liquid phase separation and biomolecular condensates. Cell 176: 419-434 (2019).

6 3. Chen, X. D. et al. Phase separation at the synapse. Nat. Neurosci. 23: 301-310 (2020).

4. Shin, Y. \& Brangwynne, C. P. Liquid phase condensation in cell physiology and disease. Science 357: eaaf4382 (2017).

5. Banani, S. F., Lee, H. O., Hyman, A. A., Rosen, M. K. Biomolecular condensates: organizers of cellular biochemistry. Nat. Rev. Mol. Cell Biol. 18: 285-298 (2017).

6. Alberti, S. \& Hyman, A. A. Biomolecular condensates at the nexus of cellular stress, protein aggregation disease and ageing. Nat. Rev. Mol. Cell Biol. 22: 196-213 (2021).

7. Wolozin, B. \& Ivanov, P. Stress granules and neurodegeneration. Nat. Rev. Neurosci. 20: 649-666 (2019).

8. Larson, A. G. et al. Liquid droplet formation by HP1 alpha suggests a role for phase separation in heterochromatin. Nature 547: 236-240 (2017).

9. Strom, A. R. et al. Phase separation drives heterochromatin domain formation. Nature 2017, 547: $241-245$ (2017).

10. Schmidt, H. B. \& Gorlich, D. Transport selectivity of nuclear pores, phase separation, and membraneless organelles. Trends Biochem. Sci. 41: 46-61 (2016).

11. Sabari, B. R. et al. Coactivator condensation at super-enhancers links phase separation and gene control. Science 361: eaar3958 (2018). 
mutation. Cell 162: 1066-1077 (2015). $301-305(2020)$

14. Setru, S. U. et al. A hydrodynamic instability drives protein droplet formation on microtubules to nucleate branches. Nat. Phys. 17: 493-498 (2021).

15. Quail, T. et al. Force generation by protein-DNA co-condensation. Nat. Phys. 17: 1007-1012 (2021). $1210(2020)$ 464-468 (2020).

18. Deviri, D. \& Safran, S. A. Physical theory of biological noise buffering by multicomponent phase separation. Proc. Natl. Acad. Sci. U.S.A. 118: e2100099118 (2021).

19. Yuan, F. et al. Membrane bending by protein phase separation. Proc. Natl. Acad. Sci. U.S.A 118: e2017435118 (2021).

20. Kanaan, N. M., Hamel, C., Grabinski, T., Combs, B. Liquid-liquid phase separation induces pathogenic tau conformations in vitro. Nat. Commun. 11: 2809 (2020).

21. Boyko, S., Surewicz, K. \& Surewicz, W. K. Regulatory mechanisms of tau protein fibrillation

22. Ray, S. et al. alpha-Synuclein aggregation nucleates through liquid-liquid phase separation. Nat. Chem. 12: 705-716 (2020).

23. Birol, M., Kumar, S., Rhoades, E., Miranker, A. D. Conformational switching within dynamic 
oligomers underpins toxic gain-of-function by diabetes-associated amyloid. Nat. Commun. 9: 1312 (2018).

24. Conicella, A. E. et al. TDP-43 alpha-helical structure tunes liquid-liquid phase separation and function. Proc. Natl. Acad. Sci. U.S.A 117: 5883-5894 (2020).

25. Taylor, J. P., Brown, R. H. \& Cleveland, D. W. Decoding ALS: from genes to mechanism. Nature 539: 197-206 (2016).

26. Ahn, J. H. et al. Phase separation drives aberrant chromatin looping and cancer development.

27. Tsang, B., Pritisanac, I., Scherer, S. W., Moses, A. M., Forman-Kay, J. D. Phase separation as a 
polyacrylamide gels. Adv. Mater. 27: 6990-6998 (2015).

35. Rosowski, K. A. et al. Elastic ripening and inhibition of liquid-liquid phase separation. Nature Physics 16: 422-425 (2020).

36. Yuan, C. Q. et al. Nucleation and growth of amino acid and peptide supramolecular polymers through liquid-liquid phase separation. Angew. Chem. Int. Ed. 58: 18116-18123 (2019).

37. Wiedenbeck, E., Kovermann, M., Gebauer, D., Colfen, H. Liquid metastable precursors of ibuprofen as aqueous nucleation intermediates. Angew. Chem. Int. Ed. 58: 19103-19109 (2019).

38. Wang, F. et al. Progress report on phase separation in polymer solutions. Adv. Mater. 31: 1806733 (2019).

39. Liu, Y.-M. et al. The possibility of changing the wettability of material surface by adjusting gravity. Research 2020: 2640834 (2020).

40. tenWolde, P. R. \& Frenkel, D. Enhancement of protein crystal nucleation by critical density fluctuations. Science 277: 1975-1978 (1997).

41. Erdemir, D., Lee, A. Y. \& Myerson, A. S. Nucleation of crystals from solution: classical and two-step models. Acc. Chem. Res. 42: 621-629 (2009).

42. Vekilov, P. G. Dense liquid precursor for the nucleation of ordered solid phases from solution. Cryst. Growth Des. 4: 671-685 (2004).

43. Yin, D. C. Protein crystallization in a magnetic field. Prog. Cryst. Growth Charact. Mater. 61: $1-26(2015)$.

44. Yin, D. C., Wakayama, N. I., Inatomi, Y., Huang W. D., Kuribayashi K. An investigation of magnetic field effects on the dissolution of lysozyme crystal and related phenomena. Acta Crystallogr. D Biol. Crystallogr. 58: 2024-2030 (2002).

45. Wang, J. et al. A molecular grammar governing the driving forces for phase sparation of prion-like 
Methods

4

Materials. Two proteins, namely lysozyme and proteinase K, were used as model proteins for the crystallization experiments. Hen egg-white lysozyme (HEWL, $6 \times$ recrystallized, Catalogue No. 100940, Seikagaku Kogyo, Japan; $3 \times$ recrystallized, Catalogue No. L4919, Sigma-Aldrich, USA), and proteinase K (Sigma-Aldrich, Catalogue No. P6556) were purchased and used directly without further purification. The other chemicals used include: $\mathrm{NaCl}$ (GR, Catalogue No. 10019308, Sinopharm Chemical Reagent Co., Ltd., China), $\mathrm{NiCl}_{2}$ (SCRC 10014218, Sinopharm Chemical Reagent Co., Ltd., China), $\mathrm{MnCl}_{2}$ (Tianjin Kemiou Chemical Reagent Co., Ltd., China), $\left(\mathrm{NH}_{4}\right)_{2} \mathrm{SO}_{4}$ (Catalogue No. 020912, Xi'an Chemical Reagent Co., Ltd., China), Tris-HCl (Catalogue No. T8230, Solarbio, Ltd., China) and sodium acetate (Catalogue No. 02112L, Xi' an Chemical Reagent Co., Ltd., China).

Gradient and localized low magnetic field generated by permanent magnet(s). We used two types of permanent magnet systems to create a gradient magnetic field, and a localized low magnetic field, respectively. The first one applied was a single cylindrical permanent magnet (Fig. 2a, made from NdFeB, S-20-10-R, purchased from Webcraft GmbH, Germany), with dimensions $\Phi 22 \mathrm{~mm} \times 11.4 \mathrm{~mm}$. This magnet was used to create a gradient magnetic field (Fig. 2c, calculated by ANSYS finite element code (ANSYS 8.0, USA)), which can be used to push objects that are more diamagnetic than the surrounding environment.

The second one applied was a pair of permanent magnets (Fig. 2b). The two permanent magnets were arranged with their $\mathrm{N}$ poles facing right to each other. As a result there was 
only a low magnetic field between the two magnets (Fig. 2d). Objects that are more

2 diamagnetic than the surrounding environment were expected to be pushed to the localized

3 low magnetic field (i.e., the central part between the two magnets).

4

\section{Model solution for observing movement of dense liquids after phase separation}

To visualize the behavior of dense liquid droplets under the influence of magnetic field, we prepared a model solution in which liquid-liquid phase separation could be observed applying a dye. The compositions of the solution: $3 \mu \mathrm{L}$ trypsin $(50 \mathrm{mg} / \mathrm{ml}), 2.8 \mu \mathrm{L}$ ammonium sulphate $(2.3 \mathrm{~mol} / \mathrm{L})$, and $1 \mu \mathrm{L} \mathrm{MnCl}_{2}(750 \mathrm{mg} / \mathrm{mL})$. All of these chemicals were purchased from Sigma-Aldrich. 0.3 - $0.5 \mu \mathrm{L}$ JBS True Blue dye (Crystal Dye, Cat. No. CO-301, Jena Bioscience, Germany) was added to the solution. The mixed solution was then sealed with paraffin oil $(4 \mu \mathrm{L})$. Liquid-liquid phase separation occur in the solution at ambient temperature (about $20^{\circ} \mathrm{C}$ ) and the usual size of the droplets was about $250-300 \mu \mathrm{m}$. Figs. 2e - $2 \mathrm{f}$ shows a number of coalescences of dense liquids under the influence of the magnetic field. Extended Data Fig. 1 shows more examples.

\section{Measurement of concentration distribution using interferometer}

The concentration distribution of protein in the magnetic field can show the effect of magnetic field on the movement of dense liquids. An increase in the concentration indicates clustering of dense liquids. To find out if the magnetic field can non-contactly cluster the dense liquids, we built a Mach-Zehnder interferometer to monitor the concentration evolution in the protein solution. Fig. $2 \mathrm{~m}$ shows the structure of the interferometer. A quartz cell (inner dimension thickness $1 \mathrm{~mm}$, width $15 \mathrm{~mm}$, and height $10 \mathrm{~mm}$ ), which was used as the container to fill 

.

with protein solution, was placed in the optical path in the interferometer. The quartz cell was inserted between the pair of cylindrical permanent magnets (as shown in Fig. 2g). In Fig. 2m, BS1 and BS2: beam splitters; MO1 and MO2: microscope objective; $\mathrm{PH} 1$ and $\mathrm{PH} 2$ : pinhole; L1 and L2: lens; M1 and M2: mirrors; TL: telecentric lens.

The protein solution conditions: $30 \mathrm{mg} / \mathrm{mL}$ hen-egg white lysozyme $(3 \times$ recrystallized, Sigma-Aldrich, Cat. No. L4919, USA) and $250 \mathrm{mg} / \mathrm{mL} \mathrm{MnCl}_{2}$ (AR, Kermel), in sodium acetate buffer at $\mathrm{pH}=4.60$. The protein concentration at five points (labeled as red dots in Fig. 2n) was monitored using the interferometer. The room temperature was controlled at $22^{\circ} \mathrm{C}$. Fig. $2 \mathrm{n}$ shows the results of the concentration evolution against the time. The results showed clear different time dependences of concentrations at the central and the surrounding points, confirming that the dense liquids were clustered at the central point (point 1).

\section{Localized protein crystallization in low magnetic field}

Protein crystallization was carried out using the same quartz cell (inner dimensions $1 \mathrm{~mm}$ $\times 15 \mathrm{~mm} \times 10 \mathrm{~mm}$ ) which was placed between the two opposite cylindrical permanent magnets as used in the interferometer measurement experiment. The crystallization conditions were: $36 \mathrm{mg} / \mathrm{mL}$ hen-egg white lysozyme (3× recrystallized, Sigma-Aldrich, Cat. No. L4919, USA; and $6 \times$ recrystallized, Catalogue No. 100940, Seikagaku Kogyo, Japan) and 250 $\mathrm{mg} / \mathrm{mL} \mathrm{MnCl}_{2}$ (AR, Kermel), in sodium acetate buffer at $\mathrm{pH}=4.60$. To observe crystallization at a lower initial concentration, the initial protein concentration was decreased to $30 \mathrm{mg} / \mathrm{mL}$. Simultaneously, crystallization outside of the magnet pair was also conducted for comparison.

After crystallization for 12 hours, the quartz cell was placed under a stereo-microscope 
1 (Olympus SZX16, Japan) and the images of the crystals were captured for subsequent

2 analysis. The results showed that protein crystallization occurs only in the region where the

3 magnetic field is low, i.e., the crystallization is localized in low magnetic field (Figs. 2i, 2k,

$421)$.

\section{Experimental platform for non-contact crystallization}

We built an experimental platform for non-contact crystallization. It includes a vertical superconducting magnet, a temperature control system, a sample holder, and a real-time monitoring system (Fig. 3a).

The superconducting magnet (JMTA-16T 50MF, Japan Superconductor Technology Co. (JASTEC), Japan) is a vertical cryogen (liquid Nitrogen \& Helium) cooled magnet working at persistent mode. The maximum field at the central axis is $16.12 \mathrm{~T}$, and the diameter of the room temperature bore is $51 \mathrm{~mm}$. Extended Data Fig. 3 shows the magnetic field $B$ distribution and the product of $B B^{\prime}$ along the vertical direction. Polyscience Inc., USA) and a copper water-jacket (home-made, with outer diameter $49 \mathrm{~mm}$, and inner diameter $43 \mathrm{~mm}$, the temperature controlled water is circulating within the thin channel with thickness of $\sim 2 \mathrm{~mm}$ ) fitted into the magnet bore (Extended Data Fig. 3). The temperature of the sample placed inside of the water jacket can be accurately controlled in the temperature range: $4-60^{\circ} \mathrm{C} \pm 0.1^{\circ} \mathrm{C}$. A similar temperature control system was placed outside 21 of the magnet. 
holder (with diameter $42.5 \mathrm{~mm}$ ) was made fit into the water jacket. A plastic vessel (bottom

diameter: $7.9 \mathrm{~mm}$, upper diameter: $8.5 \mathrm{~mm}$, height: $12 \mathrm{~mm}$ ) was used as the crystallization container, and was fixed at the upper end of the sample holder.

The real-time monitoring system (Fig. 3a, Extended Data Fig. 3) comprises $2 \mathrm{CCD}$ cameras ( $\mathrm{f}=11 \mathrm{~mm}$, Ultra Micro-CCD UN43H, ELMO Co., Japan), 2 reflecting mirrors, a ring of illuminating LEDs (light-emitting diodes, LED), and a monitoring computer. Two CCD cameras were used to capture the top- and side-view images and videos. Two reflecting mirrors were fixed in parallel at both sides of the crystallization container to guide the light for the side-view observation. The LED ring was installed below the bottom of the crystallization container, and a diffuser (diameter $42 \mathrm{~mm}$, thickness $3 \mathrm{~mm}$ ) was attached right above the LED ring to improve the homogeneity of the illuminating light. For monitoring the experiments a computer was connected with CCD cameras, and the images and videos were captured using distinct computer software (H264 WebCam Deluxe ver 4.0).

\section{Non-contact crystallization experiments in superconducting magnet}

Crystallization of two types of proteins (lysozyme and proteinase K) was carried out using the experimental platform described above.

For crystallization of lysozyme, HEWL $(6 \times$ recrystallized $)$, HEWL $(3 \times$ recrystallized $)$, $\mathrm{NaCl}$ (used as the precipitant) and $\mathrm{NiCl}_{2}$ were dissolved in sodium acetate buffer solution $(0.1$ $\mathrm{M}, \mathrm{pH} 4.6$ ) at concentrations $110 \mathrm{mg} / \mathrm{mL}, 95 \mathrm{mg} / \mathrm{mL}, 60 \mathrm{mg} / \mathrm{mL}$, and $200 \mathrm{mg} / \mathrm{mL}$, respectively.

For crystallization of proteinase $\mathrm{K}$, proteinase $\mathrm{K},\left(\mathrm{NH}_{4}\right)_{2} \mathrm{SO}_{4}$ (used as the precipitant), and $\mathrm{NiCl}_{2}$ were dissolved in the Tris- $\mathrm{HCl}$ buffer solution $(0.1 \mathrm{M}, \mathrm{pH} 8.0)$ at concentrations 37.5 $\mathrm{mg} / \mathrm{mL}, 190 \mathrm{mg} / \mathrm{mL}$, and $200 \mathrm{mg} / \mathrm{mL}$, respectively. All of the above solutions were filtered 
through a filter (Catalogue No. YA0655, Solarbio) with pore size $0.22 \mu \mathrm{m}$.

In each crystallization experiment, $100 \mu \mathrm{L}$ protein solution and $100 \mu \mathrm{L}$ precipitant solution were pipetted and mixed in the crystallization container. Then $\mathrm{NiCl}_{2}$ solution was added to the container at volumes $12,8.5$ and $9.8 \mu \mathrm{L}$ for crystallization of lysozyme $(6 \times$ recrystallized), lysozyme $(3 \times$ recrystallized $)$ and proteinase $K$, respectively. The mixed solutions were sealed using the Crystal Clear Tape (Catalogue No. HR4-506, Hampton Research, USA) and then placed in the sample holder. The crystallization temperature was controlled at $21^{\circ} \mathrm{C}$ for lysozyme, and $23^{\circ} \mathrm{C}$ for the proteinase $\mathrm{K}$.

The two CCD cameras were adjusted at appropriate focal lengths, allowing to monitor the crystallization processes occurring in the sample container. Visualizing of the crystallization process was carried for about two-days for the HEWL experiment, and one-day for the proteinase $\mathrm{K}$ experiment, for the applicable diffraction analysis. The crystallization processes were recorded and formatted into short movies using video editing software (Corel Video Studio X9).

\section{X-ray diffraction and quality analysis of protein crystals}

The crystals were harvested using nylon loop (CryoLoop, Hampton Research, USA) under the stereo-microscope, and soaked prior to shock freezing in the cryoprotectant solutions (with 20-25\% glycerol and the corresponding precipitant buffers). Then the crystals were mounted on the X-ray diffractometer (MarResearch GmbH, Norderstedt, Germany) and cryocooled in liquid nitrogen stream at $100 \mathrm{~K}$. The diffraction data were then collected. Finally, all the collected data sets were integrated and merged using the HKL-2000 software package. 
Fig. $3 \mathrm{j}$ shows the quality comparison of the crystals obtained in the non-contact

2 crystallization (labeled as "magnet" in the figure) and the control. The left panel shows the

3 comparison in resolution (for HEWL, $n=6, p=0.0004$; for proteinase $K, n=3, p=0.018$ ).

4 The middle panel shows the comparison in mosaicity. $(p=0.05$ for HEWL, and $p=0.327$ for

5 proteinase $\mathrm{K})$. The right panel shows the comparison in B factor. $(\mathrm{p}=0.079$ for HEWL, and $\mathrm{p}$

$6=0.006$ for proteinase K). The comparisons showed clear improvement in crystal quality by

7 using non-contact crystallization in the high magnetic field. 

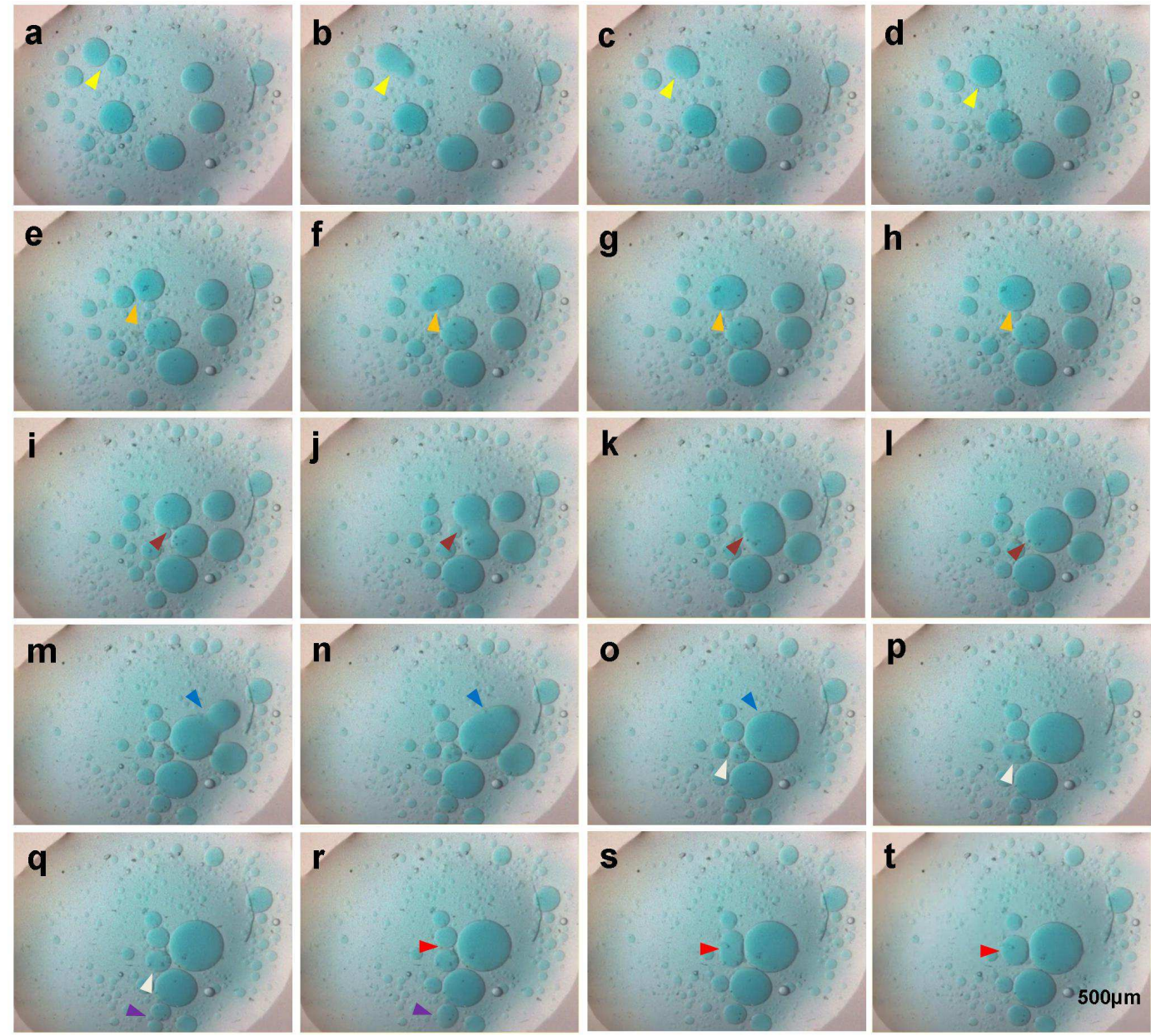

2 Extended Data Fig. 1| Evidence of non-contact manipulation of dense liquid droplets using a

3 permanent magnet. (a to t) Image series of coalescences of dense liquid droplets under the influence of

4 magnetic field. Different colored arrowheads show different coalescences. The experiment clearly

5 shows the evidence that the magnetic field can push the dense liquids together and trigger coalescence

6 of the dense liquids. 

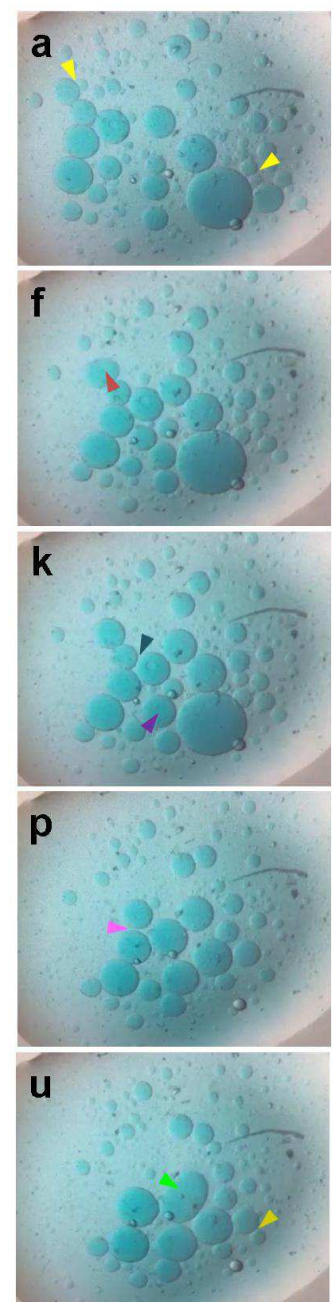
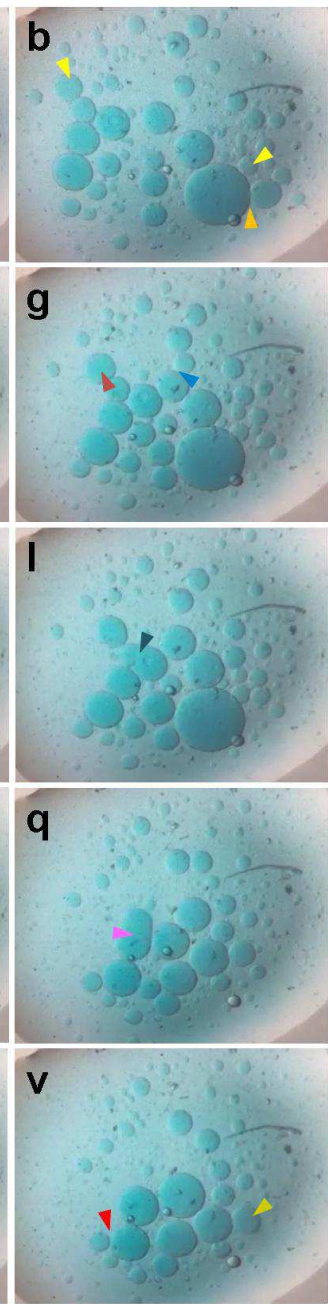
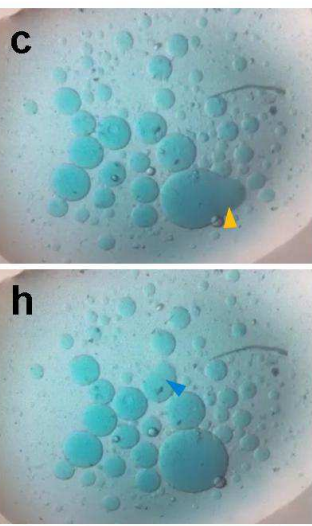

m
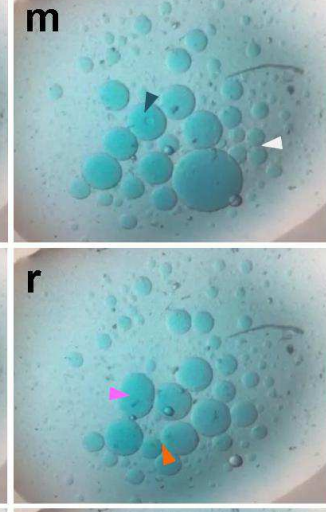

w

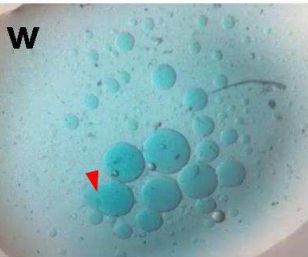

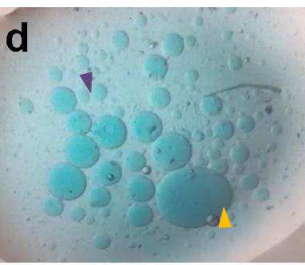

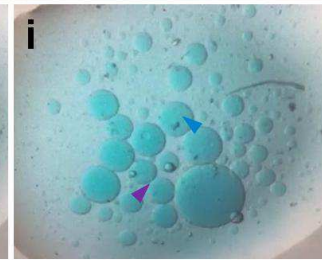

n
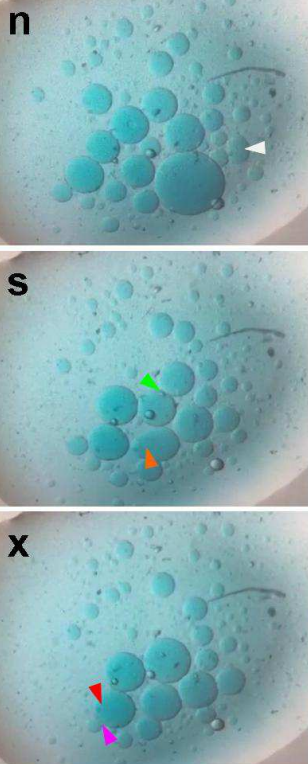
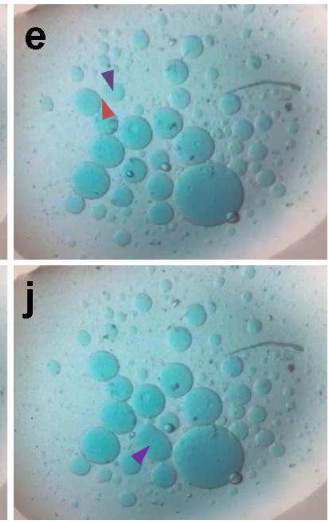

0
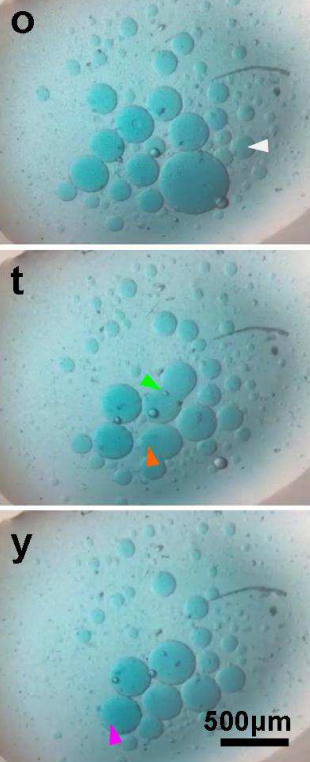

2 Extended Data Fig. 2| Evidence of non-contact manipulation of dense liquid droplets using a permanent magnet pair. A pair of cylindrical $\mathrm{NdFeB}$ permanent magnets with their $\mathrm{N}$-poles facing right

4 to each other was used. In between the N-poles, the sample with phase-separated solution was placed. magnetic field can push the dense liquids together and trigger coalescence of the dense liquids. 


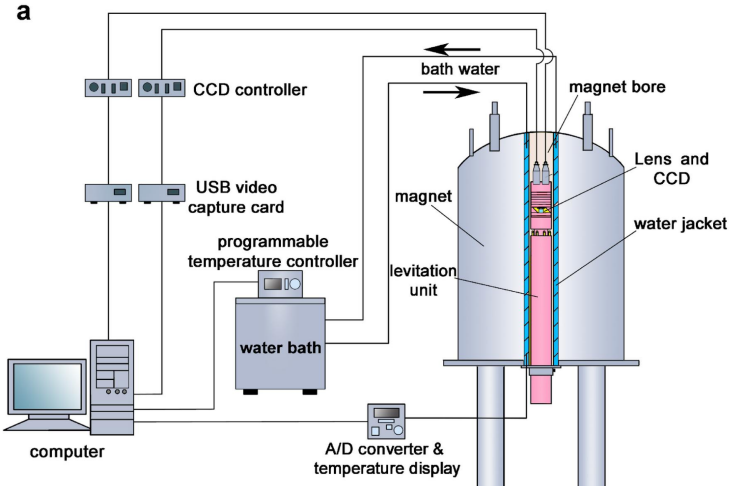

b

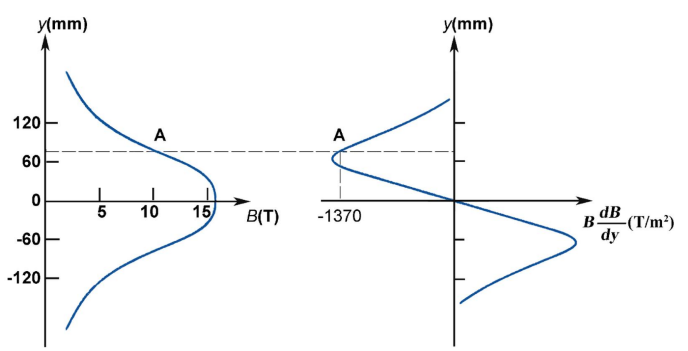

2

3 Extended Data Fig. 3| Schematic illustration of the superconducting magnet experimental platform. (a)

4 The overall experimental platform. (b) Distribution of the magnetic field $B$ and the field-gradient

5 product $B B^{\prime}$ along the vertical axis.

6 

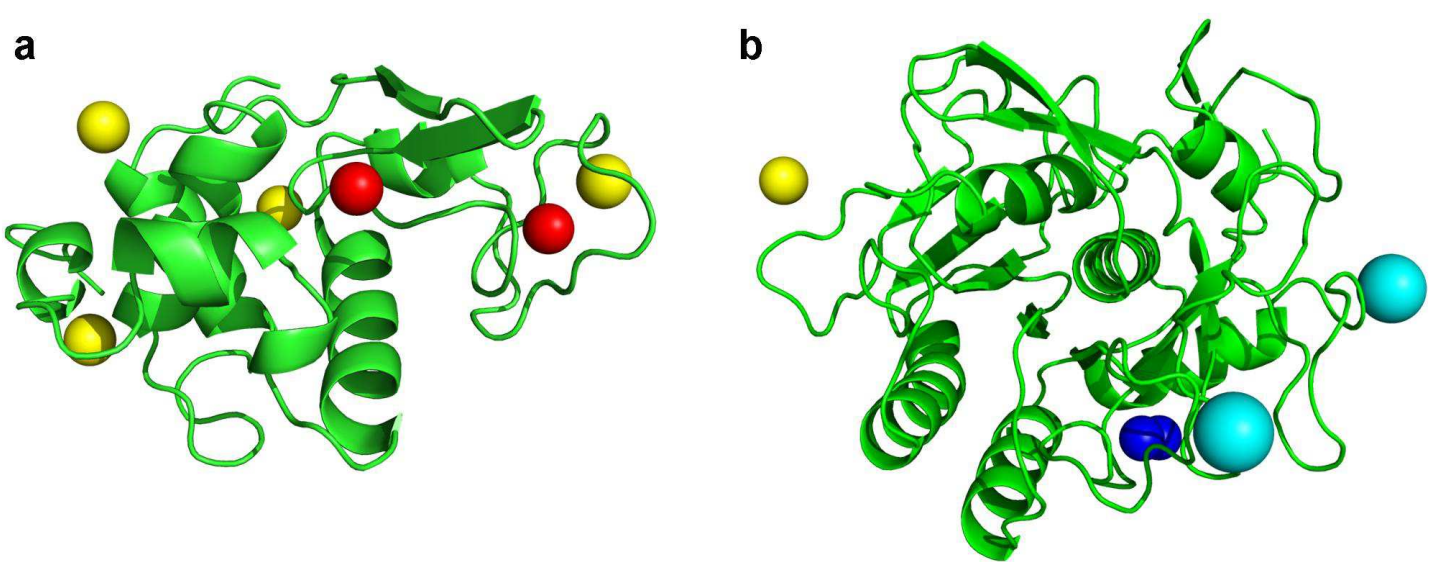

2

3 Extended Data Fig. 4| The crystal structure of lysozyme and proteinase K obtained from the

4 non-contact crystallization in the superconducting magnet. (a) The structure of lysozyme. Red beads:

$5 \mathrm{Ni}^{2+}$; Yellow beads: $\mathrm{Cl}^{-}$. (b) The structure of proteinase $\mathrm{K}$. Yellow beads: $\mathrm{Cl}^{-}$, dark blue beads: $\mathrm{Ca}^{2+}$,

6 cyan beads: $\mathrm{K}^{+}$ 


\section{Acknowledgements}

This work was supported by the National Natural Science Foundation of China (Grant No. 82172063), the Innovation Capability Support Program of Shaanxi (Program No. 2020TD-042), the National Key Research and Development Plan of China (Grant No. 2017YFA0504901), the Cluster of Excellence 'Advanced Imaging of Matter' of the Deutsche for Forschungsgemeinschaft (DFG) - EXC 2056 - Project ID 390715994, by the Helmholtz Excellence Network 'Structure, Dynamics and Control on the Atomic Scale', the DFG project BE1443/29-1, the German Aerospace Center (DLR) via project 50WB1423 and BMBF via project 05K16GUA. Partial work was carried out with the support of Shanghai Synchrotron Radiation Facility 18U1 beamline.

\section{Author contributions}

D. C. Y. conceived the research, D. C. Y, J. H. H. and C. B. designed the experiments. Z. Q. W. performed the non-contact crystallization experiments. Y. M. L. performed the concentration evolution experiment using interferometer. Q. D. C. performed the non-contact manipulation of dense liquid droplets. D. C. Y and Y. M. L. did the theoretical derivations. Y. M. L., C. Y. L., W. Y. G. and Z. Q. W. analyzed the data and prepared the figures and movies. Z. Q. W., J. J. C., and W. H. G. performed experiments using superconducting magnet. L. L. C. performed crystallization under normal conditions. Y. L. L. performed calculation of magnetic field distribution. F. Z. Z. and H. Z. performed structural determination. C. Y. Z., X. D. D., Y. J. Y. and W. J. L. discussed and analyzed the data. Z. Q. W., Y. M. L. and D. C. Y. wrote and revised the manuscript. P. S., J. H. H. and C. B. contributed materials / analysis tools. S. F., H. B., J. H. H. and C. B. discussed and revised the manuscript.. 


\section{Competing interests}

2 The authors declare no competing interests. 


\section{Supplementary Files}

This is a list of supplementary files associated with this preprint. Click to download.

- Supplementarylnformationsubmitted.pdf

- SM1.mov

- SM2.mov

- SM3.mov

- SM4.mov

- SM5.mov

- SM6.mov

- SM7.mov 